\title{
Application Educational Board Game in Senior High School English Class: A Case Study of Can Anne Go Upstairs?
}

\author{
Bin Zhu
}

\author{
College of Foreign Language, Zhejiang Normal University, Jinhua, Zhejiang, P R China (912925506@qq.com)
}

\begin{abstract}
Educational games can enhance students' motivation to learn. This article reports and reflects on the development of the educational games and board games. The author proposes a new concept educational board games which has the characteristics of both educational games and board games. By game design, test design, the author has discussed the possibility of applying educational board games into teaching. Can Anne Go upstairs? Is the first game on senior school English classroom teaching that is readily available for its description of the cards and its rule of the game. The results have put forward its framework and try to test the promotion in the classroom.
\end{abstract}

Key words — educational board games, senior high English teaching, curriculum-based game-design

\section{应用教育桌面游戏于高中英语课堂教学: 《安妮能到楼上吗?》游戏开发案例}

朱涁

浙江师范大学, 外国语学院, 金华, 浙江, 中国

摘 要 教育游戏可以有效提升学生学习的积极性。本文通过梳理教育游戏以及桌面游戏在国内外的发展情况, 笔者提出结合双 方特点的 “教育桌面游戏” 新概念。通过游戏设计、测试设计, 进一步探讨了 “教育桌面游戏” 应用在高中英语课堂教学的可能性, 并提出其开发框架。研究结果提供第一款 “教育桌面游戏 Can Anne Go upstairs?”的开发案例--包括卡牌样式、游戏规则, 并尝试在课 堂上进行测试推广。

关键词教育桌面游戏, 高中英语教学, 基于课程的游戏设计

1. 导言

教育游戏的研发早在上世纪 80 年代就受到欧美国家 教育研究者的重视, 尤其 Bowman (1984) 提出教育游戏 的模式。本世纪初教育游戏的理念来到中国, 引起了研究 的热潮。近几年来, 国内桌面游戏《三国杀》风靡一时， 掀起人们对这来自欧美的娱乐方式--桌面游戏的兴趣。通过 桌面游戏能培养人的观察能力、语言能力, 也能从中吸取 一定知识。笔者发现在中小学流行的卡片游戏就可认为是 桌面游戏的一种形式, 只是内容不符合教学所需, 那么将 教育游戏和桌面游戏结合, 内容融合高中英语课本知识, 能产生怎样的碰撞呢?

本文从教育游戏的角度出发, 提出了具有桌面游戏特
点的新型教育游戏一 “教育桌面游戏”, 阐述国内外研究现 状，提出适合高中英语教学的教育桌面游戏设计原则，给 出实际开发案例和测试方案, 尝试在高中英语课堂加以推 广。

\section{2. 文献综述}

2.1 教育游戏与教育桌面游戏的定义

在国外,教育游戏一般称为 “Edutainment”或 “Edueational Game”, 国内对于教育游戏软件,目前并没有 一个统一的定义, 纵观各类教育游戏软件的定义, 本文采用 香港学者尚俊杰、李芳乐等人所提出的观点, 认为教育游 戏本质上是教育软件, 其中的内容和任务都是与课程相关 
的, 而且它具备了电脑游戏的某些特征, 充分利用电脑游戏 的内在动机(如挑战、目标、情节、竞争、合作等)。

因为在国内尚没有研究将桌面游戏引入英语教学中, 我暂且大胆地将他们称为 “教育桌面游戏”, 指针对特定教 育目而开发能应用于辅助课堂教学的游戏, 并且区别于传 统教育游戏借助电脑或网络, 也区别于传统桌面游戏只重 视游戏体验不具有教学的针对性, 因此具有教育性和娱乐 性并重的特点, 是以游戏作为教育的手段, 设计游戏的时 候以成熟的教育理论作为理论支撑, 取得教育性和游戏性 的平衡, 发扬 “寓教于乐” 思想的新兴教育游戏, 可能成 为教育游戏的一个新分支。

\section{2.国内外研究现状综述:}

近几年来, 国内对于游戏与教育结合的研究刚刚起步, 不过全部集中在网络游戏和教育的结合。因为许多学者试 图利用游戏与教育的结合解决网络游戏带来的社会问题以 及目前教育教学上存在的种种弊端, 出现了一些较有价值 的文章。通过对相关文献查找和整理, 发现对于网络游戏 与教育这方面的研究主要开始于 2005 年, 而在 2000 年 以前几乎看不到有价值的相关文献, 同时也没有任何相关 的专著。主要是网络游戏与教育的基本理论研究与实践探 索, 桌面游戏在中国发展, 桌面游戏与教育游戏的结合, 国内教育游戏遇到的问题, 桌面游戏设计的要点这五个方 面展开。

西方在游戏进入教育的路上已经走得比较远, 这与他 们对游戏本身的研究起步较早是分不开的。早在上个世纪 80 年代初就已经有了关于教育游戏的研究。随后就有更多 的教育学者如 Driskell, Dwyer, Bracey 等人研究电视 游戏在教学中的应用, 他们认为电视游戏对教学是非常有 用的, 同时也在研究如何将电视游戏中激发内部动机国外 教育游戏研究的主要内容包括游戏理论的研究: 网络游戏 的教育价值, 教育游戏的设计、开发应用, 教育游戏的评 价体系, 教育游戏开发专业化, 教育游戏运作市场化、产 业化。

\section{3. 研究设计}

本文一定程度上也是为了解决目前我国在教育游戏上 的困局, 但整体而言, 桌面游戏在中国的发展, 还有较大 的前景可言, 桌面教育游戏作为一种新型教学工具, 仍然 具有很大的潜力。

\section{1 研究问题}

对于桌面游戏能否应用于英语课堂教学, 关键需要解 决两个问题:
什么是教育桌面游戏, 它是怎样构成的?

怎样从高中课本中寻找素材, 设计出的教育桌面游戏 案例能被学生接受吗?

\section{2 研究对象}

首先是要研究怎样的素材可以开发桌面游戏, 考虑怎 样从现在高中浙江省广泛采用的普通高中课程标准实验教 科书《英语》必修系列的课文中寻找素材, 加以开发。最 后, 研究在学生中是否适用, 笔者联系了母校浙江师范大 学附属中学的英语教师, 帮助进行实际的课堂教学, 参与 师生互动, 形成教学反馈, 不断修改设计, 形成最终的桌 面游戏卡牌。

\section{3 研究方法}

\subsection{1 教育观察实验法}

观察实验:

被试: 以方便取样的方法抽取浙江师范大学附属中学 的高一学生 80 名, 并保证男女比例平衡。

实验材料: 自编课堂行为观察表一份

工具: 人教版普通高中课程标准实验教科书《英语》 必修一第一单元配套教育桌面游戏 Can Anne go upstairs?, 并自行编写与课程相符合的教案, 详细介绍教学设计与实 施过程。

目前第一次实验采用的是《英语》必修一教育桌面游 戏 Can Anne go upstairs? 初稿

设备: 摄像机一架, 手表一个

实验程序: 将 80 名被试随机分为两组, 每组 40 名 (男 女比例平衡), 一组为实验组, 一组为对照组。实验组的学 生先进行游戏学习, 由笔者讲解游戏的具体流程并现场演 示, 保证学生对于怎么进行游戏有一定程度掌握。然后将 40 名学生分为 5 个小组, 每组 8 名学生, 进行正式游戏, 同时, 笔者在旁观察指导, 以保证游戏的顺利进行。由笔 者担任教师, 根据新的教案进行本次课堂教学, 讲授课本 知识。摄像机全程记录课堂上学生的行为表现, 在结束教 学后回放记录, 另一名英语专业学生记录在课堂行为观察 表的对应位置; 对照组由笔者担任教师, 根据传统教案进 行课堂教学, 学习与实验组相同的课本知识, 观察记录的 工作与实验组完全一致。每次实验组与对照组的教学过程 中, 将邀请一位在职高中英语教师全程听课。

实验假设:

该游戏对于学生的课堂行为 (包括学习行为与非学习 行为）有影响;

学生在课堂上的学习行为实验组多于对照组; 非学习 
行为实验组少于对照组。

的行为表现或事件，便于日后的分析总结。

课题组成员将在实验班级, 通过课堂里, 课后休息时, 近距离地观察同学们对桌面游戏的感受, 收获第一手资料。

\subsection{2 深度访谈法}

访谈法主要是针对学生参与游戏前、游戏过程中、游 戏结束后的心理感受、想法进行单个沟通, 以达到及时反馈 的作用, 通过一系列访谈活动的开展, 我们可以从中发现教 学过程中的一些问题, 发现教学过程中一些要保持的地方。

1. 访谈对象: 随机抽取观察实验中的被试, 实验组与 对照组各 10 人; 参与实验的在职英语教师。

2. 访谈目的: 了解学生对于本游戏教学的看法、体验 后感受、评价以及适应程度; 了解教师对本游戏教学的态 度、评价及接受程度。

3. 访谈内容:

实验组的被试一一游戏带来的感受, 包括进入情境、 体会角色情感的程度, 对课文的理解程度; 本游戏对学习 英语的兴趣影响程度; 新的上课方式与传统教学方式相比 的优、缺点;

对照组的被试一一对传统课堂教学形式上的看法与期 待; 对于引入游戏教学的看法、期待程度以及接受程度;

教师——对两组学生的课堂表现的评价; 对于本游戏 教学的看法; 对改进本游戏教学的意见与建议。

\section{4. 结果与讨论}

4.1 教育桌面游戏《安妮能到楼上吗?》案例设计:

笔者经过对文本的仔细研究, 提出了一下设计: 首款 教育桌面游戏《Can Anne go upstairs?》

游戏说明:

课文回顾:

在预习了第一单元的课文后, 我们对安妮的境况有了 初步的了解, 那么从安妮的日记里我们可以发现她有怎样 的经历呢, 她会被德国纳粹抓住吗?

游戏背景:

为了逃脱德国纳粹的追捕, 躲藏在荷兰阿姆斯特丹的 安妮一家有了新的麻烦, 德国纳粹得知消息, 马上要来搜 捕安妮! 情况危急的安妮一家人要尽自己的全力掩护安妮, 只要安妮能顺利逃到二楼进行躲藏, 就能避免被抓捕。轰 轰轰, 论异的事情发生了, 房间的三架楼梯各自断裂成了 六段, 怎么办? 快把楼梯搭建好吧! 安妮一家人能在德国 纳粹发现安妮之前, 顺利造好楼梯, 将安妮掩护到楼上吗?

胜利条件:

1. 安妮一家胜利: 同时满足两个条件:（1）在四个回
合内, 一家人齐心协力搭建好其中的一架楼梯。(2) 在四 个回合内安妮没有被德国纳粹抓捕。

2. 德国纳粹胜利: 满足两个条件其中的一个: (1) 在 四个回合内, 成功认出并且抓捕安妮。(2) 在四个回合内 破坏安妮一家建造楼梯。

角色:

Anne（安妮）,Father(安妮爸爸),Mother(安妮妈妈) Sister(安妮姐姐),German Nazis(德国纳粹)共三名, God(上 帝)

卡牌:

楼梯卡 18 张, 这是 3 架相同的楼梯, 每架楼梯破碎为 大小相同的 6 块, 只要在四个回合的游戏游戏者中将其中 一架楼梯建造好, 安妮就能跑到二楼, 成功逃脱。

效果卡 10 张, 分别对应不同效果

1.A deep bule sky: 出牌后, 你可以移动一张楼梯卡到 另一架楼梯。

2.The song of the birds:出牌后, 你向一名角色展示自己 的手牌。

3.Moonlight:出牌后, 你拿任一名角色的一张牌, 然后 在交给他一张。

4.Flowers: 出牌后, 令两名角色交换手牌

5.Keep Anne spellbound: 出牌后, 你指定一名角色, 他将不能再出任何牌。

6.The wind : 出牌后, 你将一张楼梯卡返回至任一名 角色。

7.The thundering: 出牌后, 你将一张楼梯卡暂时移出 游戏。

8.Dirty curtains: 出牌后, 从一名角色处抽取一张牌。

9.Dusty windows: 出牌后, 再次出一张楼梯卡, 可以 将这张楼梯卡放置在你想要的位置。

10.Face to face: 出牌后, 你观看任意一名角色的角色 卡。

角色卡 8 张: 1. Anne(安妮)2.Father(安妮爸爸)3. Mother (安妮妈妈) 4.Sister (安妮姐姐) 5.German Nazi(德国纳粹) 画像相同共三张 6.God (上帝)

时机卡 1 张: 正面印“Daytime”(白昼), 反面印“At dusk” (黄昏)

游戏流程:

1. 8 名同学为一组, 洗混角色卡后分发, 每位同学一 张, 全能的上帝亮出自己的角色卡, 并且依次 (从自己的 右手边开始) 观看其他同学的角色牌, 同时不要说任何话, 注意其他人不能亮出自己的角色卡。

2. 洗混楼梯卡和效果卡共 28 张, 由上帝平均分发给 7 名角色, 每人 4 张, 游戏开始, 由上帝右手边的角色开始 
出牌, 逆时针方向轮流出牌。此时可以出牌的时机为 “白 昼”。

出牌规则: 每名角色每回合只能出一张楼梯牌或者效 果牌。根据游戏胜利条件, 我们可以认为, 安妮一家要尽 可能的团结一致地往一个楼梯出牌, 而三位纳粹则尽量分 散出牌, 或者破坏比较完整的楼梯。

3. 当 8 名角色出完一轮牌后 (“白昼” 时机结束了), 上帝将时机卡翻面, 此时进入 “黄昏” 时机, 今天的 “建 造” 告一段落, 但是纳粹不会在这个时机休息。此时需要 所有人闭上眼睛, 全能的上帝说道: “Evil German Nazis, here your chances come! (邪恶的德国纳粹, 你们的机会来了)!” 三名纳粹睁开眼睛, 不能说话, 根据上一轮行动, 指认其 他四名角色中的一名为安妮, 若上帝作出回答: “ Justice finally beats evil!(邪不能压正啊!)” 则指认失败, 进入下一 回合。若上帝作出回答: “Poor girl,give her more time. (可 怜的女孩, 放她一条生路吧。)”纳粹第一次指认成功, 游 戏还没有结束, 此时安妮的爸爸可以亮出自己的身份牌, 把所以手牌给安妮, 代替安妮接受 “抓捕”。如果第一轮安 妮的身份就被识破, 游戏将对安妮一家非常的不利, 安妮 爸爸被抓不能出牌, 那么在接下来的一轮游戏中安妮和安 妮妈妈, 安妮姐姐如果不能成功建造一架完整的楼梯, 则 德国纳粹获胜。不论指认是否成功, 结束后所以人睁开眼 睛, 下一回合开始。

4. 在这四个回合中, 重复执行在 “白昼” 出牌, 在 “黄 昏” 抓捕的游戏规则。

\section{2 教育桌面游戏的构成体系}

开发能应用在课堂教学的桌面游戏应符合以下两个 流程:

（1）构成一套游戏卡牌的要素: 设计成型的彩色游戏 卡牌, 易于理解的游戏规则, 规则解答。

(2) 配合游戏卡牌的教案设计, 测试设计, 问卷调查, 访谈。

笔者认为并非所有课文都可以设计出一个有趣的桌面 游戏。桌面游戏设计应符合两个要素:

（1）故事性: 一般具有故事情节的文章容易设计, 原 因在于推动情节的发展相对较容易, 游戏中对抗性明显, 有正面人物, 反面人物, 或者还存在中立人物。游戏的胜 利条件明显, 正面人物胜利, 反面人物胜利, 或者中立人 物胜利。

（2）教育性: 游戏卡牌的语言要符合课文内容, 最好 原汁原味来着课文, 构建的情景必须是课文内容一致。
4.3 教育桌面游戏设计的有效性:

虽然笔者提出了桌面教育游戏的新概念, 但是它真的 有效吗? 学生会喜欢这样的形式吗? 会被教育专家认可 吗？容易被给位教学经验丰富的教师接受吗?

最终必须进行的是课堂观察实验, 实验后发放问卷, 进行深度访谈的环节。

根据大量的课堂教学反馈, 后续发放问卷调查高中生 对这款游戏的感受, 进行深入访谈, 合理修改游戏的部分 规则, 提炼出教育桌面游戏的设计原则, 尝试开创教育桌 面游戏的理论框架。

\section{5. 小结}

本次研究通过文献研究法对国内外教育游戏和桌面 游戏进行总结, 归纳各自的提点, 并结合中小学生现实, 笔者提出 “教育桌面游戏” 的新概念, 讨论桌面教育游戏 的开发, 确立课文选材, 探索设计原则, 给出设计案例-第 一款教育桌面游戏 Can Anne go upstairs?。在此基础上提出 实验测试法, 设计详细的观察实验和访谈, 方便进行进一 步的研究。

本次研究也不可避免存在这一些局限性。由于时间和 精力的限制, 设计开发的教育桌面游戏仅仅形成初稿, 有 效度不够充分, 研究深度有限。希望以后的相关研究中验 证它在教学上的作用, 进一步对教育桌面游戏进行开发, 提高普及度。

\section{参考文献(References)}

[1] Blechman, Elaine A., et al. "The Family Contract Game: Technique and Case Study." Journal of Consulting and Clinical Psychology, vol. 44, pp.449-455, 1976.

[2] Li-Chun, Wang, and Chen Ming-Puu. "The Effects Of Game Strategy And Preference-Matching On Flow Experience And Programming Performance In Game-Based Learning." Innovations In Education \& Teaching International, vol.47, pp.39-52, 2010.

[3] Petsche, Jennifer. "Engage And Excite Students With Educational Games." Knowledge Quest, vol.40, pp.42-44, 2011.

[4] Pauschenwein, J., E. Goldgruber, and A. Sfiri. "The Identification of the Potential of Game-Based Learning in Vocational Education within the Context of the Project "Play the Learning Game." International Journal of Emerging Technologies in Learning, vol.8, pp.20-23, 2013.

[5] Eisenack,Klaus,2012.A Climate Change Board Game for Interdisciplinary Communication and Education, Simulation \& Gaming, vol.44, pp.328 - 348,2012. 